\title{
SYNTHESIS AND ANTIOXIDANT EVALUATION OF NOVEL PHENOTHIAZINE LINKED SUBSTITUTEDBENZYLIDENEAMINO-1,2,4-TRIAZOLE DERIVATIVES
}

\author{
SURESH MADDILA', MEHBUB MOMIN ${ }^{1}$, SRIDEVI GORLE ${ }^{3}$, LAVANYA PALAKONDU $^{*}$ AND \\ SREEKANTH B JONNALAGADDA ${ }^{1}$
}

\begin{abstract}
${ }^{I}$ School of Chemistry \& Physics, University of KwaZulu-Natal, Westville Campus, Chiltern Hills, Private Bag 54001, Durban-4000, South Africa.
${ }^{2}$ Department of Chemistry, Annamacharya Institute of Technology \& Sciences J.N.T.University, Tirupati -517 502, Andhra Pradesh, India.

${ }^{3}$ Discipline of Biochemistry, School of Life Sciences, University of KwaZulu-Natal, Westville Campus, Chilten Hills, Durban-4000, South Africa.
\end{abstract}

\begin{abstract}
A series of novel 5-((10H-phenothiazin-10yl)methyl)-4-(substitutedbenzylideneamino)-4H-1,2,4-triazole-3-thiol derivatives (6a-i) have been synthesized from compound (1) through a multi-step reaction. The key intermediate (5) afforded a series of title compounds (6a-i) on condensation with various suitable aldehydes in the presence of $\mathrm{H}_{2} \mathrm{SO}_{4}$. The structures of novel compounds were characterized based on their elemental analysis, IR, ${ }^{1} \mathrm{H}-\mathrm{NMR},{ }^{13} \mathrm{C}-\mathrm{NMR}$ and $\mathrm{MS}$ spectral data. All these novel compounds were screened for their in vitro antioxidant activity by employing nitric oxide, hydrogen peroxide, and DPPH radical scavenging assays. The compounds $\mathbf{6 d}, \mathbf{6 e}$ and $\mathbf{6 i}$ demonstrated potent antioxidant activity as these contain the electron-releasing groups.
\end{abstract}

Keywords: Synthesis, Anti-oxidant activity, 1,2,4-Triazole, Phenothiazines.

\section{INTRODUCTION}

The heterocyclic compounds chemistry continues to be an emerging field in the organic and pharmaceutical chemistry ${ }^{1}$. Heterocyclic compounds are present in various drugs, several natural products, some vitamins, biomolecules, and biologically active compounds such as anti-inflammatory, antitumour, antimalarial, antidepressant, anti-HIV, antibiotic and antimicrobial agents etc $^{2,3}$. Nowadays, antioxidants provoke researcher's interest in both medicinal plants and synthetic compounds. Antioxidants (natural or synthetic) are the molecules, which are able to neutralize the free radicals by acting at various stages like interception, prevention and repair ${ }^{4,5}$. It is therefore necessary to develop therapeutic agents with improved potential for treating broadspectrum of oxidant infections. Vast data suggests that the free radical-reactive nitrogen and oxygen species influence on the damage of biomolecules, which will further lead to etiology of many human diseases ${ }^{6}$. The high resistance acquired by microbes against the antioxidant drugs existing in the market is of a great challenge to the scientific organizations, involved in the development of novel and effective drugs against human diseases? ${ }^{7}$. Synthesis of nitrogen and sulfur bearing heterocyclic derivatives has been the great interest of researchers for the past few decades, due to their potential use in the pharmaceutical and medicinal applications ${ }^{8,9}$.

Phenothiazines are heterocyclic molecules with two benzene rings associated to the tricyclic system via nitrogen, sulfur atoms. Phenothiazine occurs in various antipsychotic and antihistaminic drugs ${ }^{10,}{ }^{11}$. These phenothiazine moieties are widely employed as antibacterial ${ }^{12}$, antiviral ${ }^{13}$, anti-inflammatory ${ }^{14,15}$, anticancer ${ }^{16,17}$, tranquilizers agents ${ }^{18,19}$, antitubercular ${ }^{20}$, anticonvulsant ${ }^{21}$, antiproliferative ${ }^{22}$ and as anti-HIV agents ${ }^{23}$. Triazoles and their derivatives established a main class of organic molecules in a broad spectrum of biological activities, various industrial, agricultural and pharmaceutical fields ${ }^{24-26}$. Triazole ring derivatization lies on the 'bioisosterism phenomenon' in which oxygen of oxadiazole nucleus is replaced with the nitrogen of triazole analogue ${ }^{27}$. Triazole derivatives are the therapeutically fascinating drug candidates including antifunga ${ }^{28}$, antioxidant ${ }^{29}$, antibacterial ${ }^{30}$, insecticidal ${ }^{31}$, anti-inflammatory ${ }^{15}$, antineoplastic ${ }^{32}$, antiviral ${ }^{33}$, sedatives $^{34}$, anticonvulsant ${ }^{35,36}$, anti-histaminic ${ }^{27,32}$, antitumor ${ }^{37}$, and CNS stimulants ${ }^{38}$ etc. $^{34}$

In continuation of our research in the domain of biologically active molecules ${ }^{15,27-31,39-43}$, the novel phenothiazine and triazole derivatives, in combination, were synthesized with the hope that these derivatives may exhibit a synergistic effect for the antioxidant activity.

\section{RESULTS AND DISCUSSION}

Chemistry

A few procedures have been reported in the literature for the synthesis of triazole bearing phenothiazines derivatives and their antimicrobial activity. Literature survey shows quite many reaction methods were reported for synthesis of various triazole bearing phenothiazines derivatives. To mention a few, such protocols employed microwave irradiation [44] and microwave and catalyst [45]. These methods have some limitations and drawbacks, such as use of toxic reagents, strong acidic or basic conditions, costly reagents and catalysts, strict reaction conditions, tedious steps, low product yields and/or long reaction times, which restrict their scope in practical applications. Therefore, a novel protocol with good and inexpensive catalyst demanding less reaction times is sought after. The method for the synthesis of triazole bearing phenothiazines is described in Scheme 1. By adapting our previously described methods ${ }^{15}$, the compound $10 \mathrm{H}$-phenothiazine was preserved with chloroacetate 2 with $\mathrm{K}_{2} \mathrm{CO}_{3}$ catalyst present in acetone solvent medium to give ethyl 2-(10H-phenothiazin10-yl)acetate (3). Further, the compound was reacted with $\mathrm{KOH}$ as a basic media to afford 2-(10H-phenothiazin-10-yl) acetic acid (4). The compound 4 was treated under solvent and catalyst free conditions with thiocarbohydrazide to obtain the cyclic triazole compound (5). Next, substituted aldehydes were treated with compound 5 in the presence of concentrated $\mathrm{H}_{2} \mathrm{SO}_{4}$ as a catalyst in DMF solvent media to attain the proposed title (5- $((10 H$-phenothiazin10yl)methyl)-4-(substitutedbenzylideneamino)-4H-1,2,4-triazole-3-thiol (6ai) derivatives in good to high yields (Scheme 1). The characterization of the synthesized title compounds was executed by FT-IR, ${ }^{1} \mathrm{H}-\mathrm{NMR},{ }^{13} \mathrm{C}-\mathrm{NMR}$ and LC-MS spectral analysis.

All the analysed compounds gave satisfactory analyses for the anticipated structures, which were established by their spectral data. The ${ }^{1} \mathrm{H}-\mathrm{NMR}$ spectra of the compound (3) demonstrated distinctive peaks in the range of triplet at $\delta$ $1.32 \mathrm{ppm}$ for $\mathrm{CH}_{2} \mathrm{CH}_{3}$, quartet at $\delta 4.24 \mathrm{ppm}$ for $\mathrm{CH}_{2} \mathrm{CH}_{3}$ and singlet at $\delta 4.84$ ppm due to $\mathrm{CH}_{2} \mathrm{CO}$. The IR spectra of the compound (3) exhibited absorption bands at $1576,1609,1672,2832$ and $3378 \mathrm{~cm}^{-1}$ corresponding to the stretching frequencies of, $\mathrm{C}=\mathrm{N}, \mathrm{C}=\mathrm{C}, \mathrm{C}=\mathrm{O}, \mathrm{CH}$ and $\mathrm{COOH}$ groups, respectively. The ${ }^{1} \mathrm{H}-\mathrm{NMR}$ spectra of the compound (4) exhibited a singlet for the two protons of the $\mathrm{CH}_{2} \mathrm{CO}$ group at $\delta=4.35 \mathrm{ppm}$ and a broad singlet at $\delta 11.49 \mathrm{ppm}$ due to $\mathrm{COOH}$ proton. IR spectra of compound (5) showed characteristic absorption bands at $1571,1658,2762,2894$, and $3458 \mathrm{~cm}^{-1}$ for the $\mathrm{C}=\mathrm{N}, \mathrm{C}=\mathrm{O}, \mathrm{SH}, \mathrm{CH}$ and $\mathrm{NH}_{2}$, stretching vibrations, respectively. Its ${ }^{1} \mathrm{H}-\mathrm{NMR}$ spectra showed a broad singlet for the one proton of the SH group at $\delta 12.53 \mathrm{ppm}$, while the $\mathrm{NH}_{2}$ group appeared as a singlet for the two protons at $\delta 5.67 \mathrm{ppm}$ and singlet at 5.30 ppm due to the $\mathrm{NCH}_{2}$ group respectively.

The title derivatives (6a-i) were assigned on the basis of spectral analysis. The IR spectra of the title compounds exhibited very similar features and showed the absorption bands range at $2888-2898 \mathrm{~cm}^{-1}, 2758-2770 \mathrm{~cm}^{-1}$, $1650-1658 \mathrm{~cm}^{-1}$ and $1570-1578 \mathrm{~cm}^{-1}$ for the $\mathrm{CH}, \mathrm{SH}, \mathrm{C}=\mathrm{O}$ and $\mathrm{C}=\mathrm{N}$ stretching vibrations. The physical data, ${ }^{1} \mathrm{H}-\mathrm{NMR},{ }^{13} \mathrm{C}-\mathrm{NMR}, \mathrm{LC}-\mathrm{MS}$ and elemental spectral data for all the synthesized compounds are reported in experimental protocols.

Antioxidant testing

The synthesized novel compounds $\mathbf{6 a - i}$ were evaluated for in vitro antioxidant activity by $\mathrm{NO}, \mathrm{H}_{2} \mathrm{O}_{2}$, DPPH methods and the results are tabulated 
in Table 1, 2 and 3 respectively. Among all the compounds screened for antioxidant activity, the compounds $\mathbf{6 d}, \mathbf{6 e}, \mathbf{6 i}$ showed potential antioxidant activity as compared to the ascorbic acid (Reference drug) in all three methods employed. This potential activity by compounds $\mathbf{6 d}, \mathbf{6 e}, \mathbf{6 i}$ can be ascribed to the presence of mild electron releasing groups (methoxy, dimethoxy and hydroxyl) appended to the aromatic benzene rings. However, the compounds $\mathbf{6 a}, \mathbf{6 b}, \mathbf{6 c}, \mathbf{6 f}, \mathbf{6 g}$ and $\mathbf{6 h}$ revealed moderate antioxidant activity. Results suggest that methoxy, dimethoxy and hydroxyl substituted compounds (6d, 6e, 6i) offered better antioxidant activity when compared to that of the compounds $\mathbf{6 a}, \mathbf{6 b}, \mathbf{6 c}, \mathbf{6 f}, \mathbf{6 g}$ and $\mathbf{6 h}$ with electron-withdrawing groups (halo). The $\mathrm{IC}_{50}$ value of the standard ascorbic acid using the NO method was found to be 15.70 $\mu \mathrm{g} / \mathrm{mL}$ at $25 \mu \mathrm{g} / \mathrm{mL}$ whereas the $\mathrm{IC}_{50}$ values of the compounds $\mathbf{6 e}, \mathbf{6 i}$ and $\mathbf{6 d}$ were found to be $16.98,17.54$ and $17.91 \mu \mathrm{g} / \mathrm{mL}$, respectively. More, the tables $\mathbf{1}, \mathbf{2}$ and $\mathbf{3}$ designates that the antioxidant activity in $\mathrm{NO}, \mathrm{DPPH}$ and $\mathrm{H}_{2} \mathrm{O}_{2}$ methods which increased with concentration.

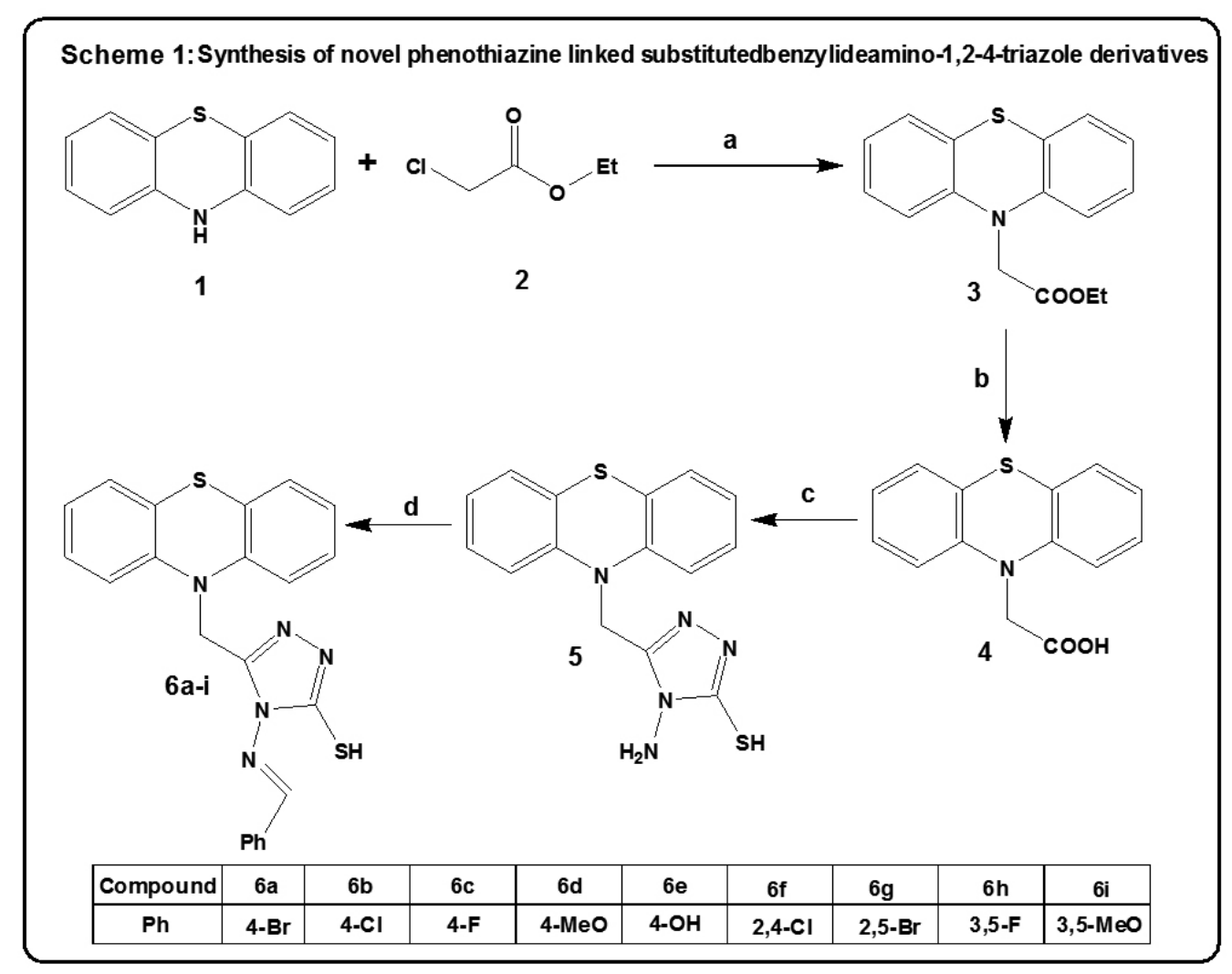

Reagents \& Conditions: (a) $\mathrm{K}_{2} \mathrm{CO}_{3}, 78^{\circ} \mathrm{C}$, reflux, 3-4 h; (b) $\mathrm{KOH}, \mathrm{R} . \mathrm{T}$., 12 h; (c) thiocarbohydrazide, heat, 4-5 h; (d) substituted aldehydes, Con $\mathrm{H}_{2} \mathrm{SO}_{4}, \mathrm{DMF}, 25^{\circ} \mathrm{C}, 5-6 \mathrm{~h}$.

Table 1: In vitro antioxidant activity of compounds (6a-i) determined by NO method

\begin{tabular}{|c|c|c|c|c|c|}
\hline \multirow[t]{2}{*}{ Compound } & \multicolumn{4}{|c|}{ Concentration $(\mu \mathrm{g} / \mathrm{mL})$} & $\mathrm{IC}_{50}$ \\
\hline & 25 & 50 & 75 & 100 & \\
\hline $6 \mathbf{a}$ & $68.41 \pm 0.90$ & $72.59 \pm 1.37$ & $74.18 \pm 0.94$ & $82.40 \pm 1.23$ & $19.52 \pm 1.04$ \\
\hline $6 \mathbf{b}$ & $60.97 \pm 1.41$ & $63.37 \pm 1.30$ & $67.65 \pm 1.24$ & $72.35 \pm 0.80$ & $20.49 \pm 0.95$ \\
\hline $6 c$ & $62.73 \pm 1.17$ & $67.48 \pm 1.24$ & $69.94 \pm 0.88$ & $73.16 \pm 0.95$ & $21.01 \pm 0.70$ \\
\hline 6d & $75.02 \pm 0.22$ & $78.14 \pm 0.45$ & $85.45 \pm 0.61$ & $84.88 \pm 0.76$ & $17.91 \pm 0.86$ \\
\hline $6 e$ & $78.18 \pm 0.30$ & $83.25 \pm 0.49$ & $86.60 \pm 0.71$ & $86.15 \pm 0.78$ & $16.98 \pm 0.69$ \\
\hline $6 f$ & $65.37 \pm 1.17$ & $70.22 \pm 1.57$ & $75.69 \pm 1.41$ & $80.53 \pm 0.71$ & $20.41 \pm 1.24$ \\
\hline $6 \mathrm{~g}$ & $73.90 \pm 0.86$ & $75.06 \pm 0.94$ & $79.25 \pm 1.06$ & $83.36 \pm 1.41$ & $18.14 \pm 0.57$ \\
\hline $6 h$ & $54.64 \pm 1.38$ & $57.49 \pm 1.24$ & $61.03 \pm 0.71$ & $65.28 \pm 1.08$ & $24.30 \pm 1.08$ \\
\hline $6 \mathbf{i}$ & $71.26 \pm 0.84$ & $74.84 \pm 1.05$ & $79.14 \pm 1.40$ & $84.93 \pm 1.26$ & $17.54 \pm 0.93$ \\
\hline Ascorbic acid* & $85.76 \pm 0.13$ & $84.92 \pm 0.38$ & $87.32 \pm 0.55$ & $91.42 \pm 0.68$ & $15.70 \pm 0.54$ \\
\hline Control & - & - & - & - & - \\
\hline
\end{tabular}

(-) Showed no scavenging activity. Values were the means of three replicates \pm SD.

* = Standard 
Table 2: In vitro antioxidant activity of compounds (6a-i) determined by $\mathrm{H} 2 \mathrm{O} 2$ method.

\begin{tabular}{|c|c|c|c|c|c|}
\hline Compound & \multicolumn{5}{|c|}{ Concentration $(\boldsymbol{\mu g} / \mathbf{m L})$} \\
\hline & $\mathbf{2 5}$ & $\mathbf{5 0}$ & $\mathbf{7 5}$ & $\mathbf{1 0 0}$ & IC $_{\mathbf{5 0}}$ \\
\hline $\mathbf{6 a}$ & $61.27 \pm 1.07$ & $64.49 \pm 1.28$ & $68.84 \pm 1.58$ & $71.93 \pm 0.83$ & $21.74 \pm 0.53$ \\
\hline $\mathbf{6 b}$ & $52.36 \pm 1.17$ & $55.03 \pm 0.87$ & $59.12 \pm 0.96$ & $64.72 \pm 0.63$ & $25.33 \pm 1.05$ \\
\hline $\mathbf{6 c}$ & $55.51 \pm 1.37$ & $58.31 \pm 1.22$ & $61.11 \pm 0.71$ & $64.27 \pm 1.08$ & $26.10 \pm 0.97$ \\
\hline $\mathbf{6 d}$ & $64.88 \pm 0.31$ & $66.50 \pm 0.47$ & $69.97 \pm 0.66$ & $73.52 \pm 0.77$ & $20.57 \pm 1.25$ \\
\hline $\mathbf{6 e}$ & $67.29 \pm 1.14$ & $70.52 \pm 1.30$ & $72.68 \pm 0.61$ & $75.19 \pm 0.71$ & $19.85 \pm 0.61$ \\
\hline $\mathbf{6 f}$ & $54.09 \pm 0.89$ & $57.60 \pm 1.38$ & $60.95 \pm 0.79$ & $65.20 \pm 1.05$ & $24.54 \pm 0.41$ \\
\hline $\mathbf{6 g}$ & $57.68 \pm 1.14$ & $61.48 \pm 1.19$ & $68.12 \pm 1.47$ & $70.93 \pm 1.54$ & $21.33 \pm 1.07$ \\
\hline $\mathbf{6 h}$ & $47.08 \pm 0.87$ & $49.37 \pm 1.16$ & $52.44 \pm 1.26$ & $55.62 \pm 1.38$ & $27.76 \pm 0.64$ \\
\hline $\mathbf{6 i}$ & $63.07 \pm 0.81$ & $66.84 \pm 1.58$ & $70.22 \pm 1.06$ & $72.59 \pm 1.37$ & $20.47 \pm 1.24$ \\
\hline Ascorbic acid* & $77.42 \pm 0.17$ & $79.61 \pm 0.34$ & $83.39 \pm 0.63$ & $87.59 \pm 0.70$ & $17.35 \pm 0.28$ \\
\hline Control & - & - & - & - & - \\
\hline
\end{tabular}

(-) Showed no scavenging activity. Values were the means of three replicates \pm SD.

* = Standard

Table 3: In vitro antioxidant activity of compounds (6a-i) determined by DPPH method.

\begin{tabular}{|c|c|c|c|c|c|}
\hline Compound & \multicolumn{5}{|c|}{ Concentration $(\boldsymbol{\mu g} / \mathbf{m L})$} \\
\hline & $\mathbf{2 5}$ & $\mathbf{5 0}$ & $\mathbf{7 5}$ & $\mathbf{1 0 0}$ & $\mathbf{I C}_{50}$ \\
\hline $\mathbf{6 a}$ & $64.87 \pm 0.71$ & $68.93 \pm 1.32$ & $72.48 \pm 1.29$ & $77.64 \pm 1.34$ & $20.57 \pm 1.23$ \\
\hline $\mathbf{6 b}$ & $61.27 \pm 1.07$ & $64.49 \pm 1.28$ & $68.84 \pm 1.58$ & $71.93 \pm 0.83$ & $21.74 \pm 0.53$ \\
\hline $\mathbf{6 c}$ & $54.75 \pm 1.74$ & $60.25 \pm 1.05$ & $63.37 \pm 0.92$ & $67.81 \pm 1.58$ & $25.70 \pm 1.47$ \\
\hline $\mathbf{6 d}$ & $71.30 \pm 1.06$ & $75.56 \pm 0.88$ & $75.87 \pm 1.30$ & $82.28 \pm 1.00$ & $18.48 \pm 1.04$ \\
\hline $\mathbf{6 e}$ & $78.43 \pm 0.28$ & $81.64 \pm 0.48$ & $85.28 \pm 0.62$ & $84.76 \pm 0.78$ & $17.88 \pm 0.28$ \\
\hline $\mathbf{6 f}$ & $63.04 \pm 1.40$ & $67.83 \pm 1.56$ & $71.92 \pm 0.78$ & $74.89 \pm 1.07$ & $21.14 \pm 0.96$ \\
\hline $\mathbf{6 g}$ & $65.70 \pm 1.44$ & $69.41 \pm 1.21$ & $74.84 \pm 1.56$ & $78.53 \pm 0.96$ & $20.30 \pm 1.12$ \\
\hline $\mathbf{6 h}$ & $49.65 \pm 0.60$ & $53.86 \pm 1.24$ & $57.63 \pm 0.55$ & $62.93 \pm 0.82$ & $26.69 \pm 1.10$ \\
\hline $\mathbf{6 i}$ & $76.46 \pm 0.30$ & $73.83 \pm 0.66$ & $74.36 \pm 0.76$ & $89.13 \pm 0.80$ & $18.08 \pm 0.51$ \\
\hline Ascorbic acid* & $84.68 \pm 0.18$ & $83.52 \pm 0.32$ & $88.52 \pm 0.43$ & $86.22 \pm 0.54$ & $16.10 \pm 0.45$ \\
\hline Control & - & - & - & - & - \\
\hline
\end{tabular}

(-) Showed no scavenging activity. Values were the means of three replicates \pm SD.

\section{CONCLUSION}

In the current study, we have developed the synthesis of 9 new derivatives of novel phenothiazine linked substitutedbenzylideneamino-1,2,4-triazole derivatives (6a-i). All have been characterized by spectral studies. In vitro, their antioxidant activity was also investigated by employing nitric oxide, hydrogen peroxide, and DPPH radical scavenging assays. The evaluation of antioxidant screening data reveals that among the 9 compounds screened, compounds $\mathbf{6 d}, \mathbf{6 e} \& \mathbf{6} \mathbf{i}$ showed potent antioxidant activity almost equivalent to that of standards.

\section{EXPERIMENTAL}

All chemicals and reagents required for the reaction were of analytical grade and were used without any further purification. Melting points were recorded on a Buchi Melting Point B-545 apparatus. Bruker AMX $400 \mathrm{MHz}$ NMR spectrometer was used to record the ${ }^{1} \mathrm{H}$ NMR and ${ }^{13} \mathrm{C}$ NMR $(400 \mathrm{MHz})$ spectral values. The $\mathrm{CDCl}_{3} / \mathrm{DMSO}-\mathrm{d}_{6}$ solution was utilized for this while TMS served as the internal standard. TMS was further used as an internal standard for reporting the all chemical shifts in $\delta(\mathrm{ppm})$. The FT-IR spectrum for the samples was established using a Perkin Elmer Perkin Elmer Precisely 100 FT-
IR spectrometer at the $400-4000 \mathrm{~cm}^{-1}$ area. LCMS spectral data was recorded on a MASPEC low resolution mass spectrometer operated at $70 \mathrm{eV}$. Purity of all the reaction products was confirmed by TLC using aluminum plates coated with silica gel (Merck Kieselgel 60 F254).

Synthesis of ethyl 2-(10H-phenothiazin-10-yl)acetate (3)

To a solution of $10 \mathrm{H}$-phenothiazine $\mathbf{1}(1 \mathrm{mmol})$ in acetone $(20 \mathrm{~mL})$ was added $\mathrm{K}_{2} \mathrm{CO}_{3}(3 \mathrm{mmol})$ at $25^{\circ} \mathrm{C}$ followed by the addition of ethyl chloroacetate $2(1.2 \mathrm{mmol})$ with reflux at $78^{\circ} \mathrm{C}$ for $3-4 \mathrm{~h}$. The completion of reaction was monitored by TLC. Next, the reaction mixture was cooled, poured into icewater and was extracted using ethyl acetate solution. Further, the organic layer was evaporated and recrystalized to obtain ethyl 2-(10H-phenothiazin-10-yl) acetate as solid.

Synthesis of 2-(10H-phenothiazin-10-yl)acetic acid (4)

The solutions of compound $3(1 \mathrm{mmol})$, dissolved in ethanol $(10 \mathrm{~mL})$ and potassium hydroxide $(3 \mathrm{mmol})$ in water $(5 \mathrm{~mL})$ were combined and the mixture was left for stirring for $12 \mathrm{~h}$ at $25{ }^{\circ} \mathrm{C}$. The reaction was monitored by TLC for completion. The reaction mixture was then transferred into cold water and acidified with $\mathrm{HCl}$ to obtain a solid product by filtration. Finally, the crude was recrystallized with ethyl acetate to afford a pure compound 2-(10H-phenothiazin-10-yl)acetic acid.

General procedure for the synthesis of 5-((10H-phenothiazin-10-yl) 
methyl)-4-amino-4H-1,2,4-triazole-3-thiol (5)

A mixture of compound 4 (1 mmol) and thiocarbohydrazide $(1 \mathrm{mmol})$ was heated under solvent-free conditions for $4-5 \mathrm{~h}$ and the reaction end-point was examined by TLC. The reaction mixture was left to cool for $1 \mathrm{~h}$ and subsequently excess acid compound was removed using sodium bicarbonate solution. Thereafter, the above mixture was filtered, dried and finally recrystallized with DMF to achieve compound 5.

Synthesis of 5-((10H-Phenothiazin-10yl)methyl)-4(substitutedbenzylideneamino)-4H-1,2,4-triazole-3-thiol (6a-i)

An equimolar ratio of compound $\mathbf{5}$ and substituted aldehydes (1x1), a few drops of concentrated $\mathrm{H}_{2} \mathrm{SO}_{4}$ in DMF $(10 \mathrm{~mL})$ solvent were stirred at 25 ${ }^{\circ} \mathrm{C}$ for $5-6 \mathrm{~h}$. After this, the reaction mixture was poured into ice-cold water, filtered to collect the solid product and was dried. The resultant crude was then recrystallized with ethyl acetate to accomplish the title compounds as excellent yields $(6 \mathbf{6}-\mathbf{i})$

5-((10H-Phenothiazin-10yl)methyl)-4-(4-bromobenzylideneamino)-4H1,2,4-triazole-3-thiol (6a)

Yield 80\%; m.p. $222-224{ }^{\circ} \mathrm{C}$; IR $(\mathrm{KBr}) v\left(\mathrm{~cm}^{-1}\right)$ : $2892(\mathrm{CH}), 2761(\mathrm{SH})$, $1654(\mathrm{C}=\mathrm{O}), 1570(\mathrm{C}=\mathrm{N}) 720(\mathrm{C}-\mathrm{Br}) ;{ }^{1} \mathrm{H}$ NMR $(\mathrm{DMSO}-d, 400 \mathrm{MHz}) \delta: 12.50$ $(\mathrm{s}, 1 \mathrm{H}, \mathrm{SH}), 9.30(\mathrm{~s}, 1 \mathrm{H}), 7.71(\mathrm{dd}, 2 \mathrm{H}, \mathrm{Ar}-\mathrm{H}, J=8.01,2.51 \mathrm{~Hz}), 7.59(\mathrm{dd}, 2 \mathrm{H}$ $J=8.05,2.49$, Ar-H), 7.28 (m, 4H, Ar-H), 7.11 (dd, 2H, Ar-H, J=7.95, 2.36 $\mathrm{Hz}), 7.05\left(\mathrm{dd}, 2 \mathrm{H}, J=7.80,2.10 \mathrm{~Hz}\right.$ ), $5.20\left(\mathrm{~s}, 2 \mathrm{H}, \mathrm{NCH}_{2}\right) ;{ }^{13} \mathrm{C}$ NMR (DMSO$d, 100 \mathrm{MHz}) \delta: 165.10,158.18,154.20,144.12(2 \mathrm{C}), 131.20$ (2C), 130.30 , 128.90 (2C), 128.10 (2), 127.62 (2C), 125.32, 123.24 (2C), 122 (2C), 115.67 (2C), 55.14; LC-MS $(70 \mathrm{eV}): \mathrm{m} / \mathrm{z}=495$. Anal. Calcd. for $\mathrm{C}_{22} \mathrm{H}_{16} \mathrm{BrN}_{5} \mathrm{~S}_{2}: \mathrm{C}$, 53.44; H, 3.26; N, 14.16. Found: C, 53.47; H, 3.29; N, 14.19.

5-((10H-Phenothiazin-10yl)methyl)-4-(4-chlorobenzylideneamino)-4H1,2,4-triazole-3-thiol (6b)

Yield 70\%; m.p. $208-210{ }^{\circ} \mathrm{C}$; IR (KBr)v $\left(\mathrm{cm}^{-1}\right)$ : $2893(\mathrm{CH}), 2768(\mathrm{SH})$, $1650(\mathrm{C}=\mathrm{O}), 1574(\mathrm{C}=\mathrm{N}) 680(\mathrm{C}-\mathrm{Cl}) ;{ }^{1} \mathrm{H}$ NMR (DMSO-d, $\left.400 \mathrm{MHz}\right) \delta: 12.40$ $(\mathrm{s}, 1 \mathrm{H}, \mathrm{SH}), 9.35(\mathrm{~s}, 1 \mathrm{H}), 7.77(\mathrm{dd}, 2 \mathrm{H}, \mathrm{Ar}-\mathrm{H}, J=8.05,2.55 \mathrm{~Hz}), 7.54(\mathrm{dd}, 2 \mathrm{H}$, $J=8.10,2.48, \mathrm{Ar}-\mathrm{H}), 7.22(\mathrm{~m}, 4 \mathrm{H}, \mathrm{Ar}-\mathrm{H}), 7.15$ (dd, 2H, Ar-H, $J=7.95,2.36$ $\mathrm{Hz}), 7.01\left(\mathrm{dd}, 2 \mathrm{H}, J=7.85,2.10 \mathrm{~Hz}\right.$ ), $5.10\left(\mathrm{~s}, 2 \mathrm{H}, \mathrm{NCH}_{2}\right) ;{ }^{13} \mathrm{C} \mathrm{NMR}$ (DMSO$\left.d_{6}, 100 \mathrm{MHz}\right) \delta: 165.15,158.22,154.28,144.25$ (2C), 135.40, 131.20, 130.80 (2C), 128.70 (2C), 128.12 (2), 127.23 (2C), 123.22 (2C), 122.50 (2C), 115.60 (2C), 55.14; LC-MS $(70 \mathrm{eV}): \mathrm{m} / \mathrm{z}=449.05$. Anal. Calcd. for $\mathrm{C}_{22} \mathrm{H}_{16} \mathrm{ClN}_{5} \mathrm{~S}_{2}$ : C, 58.72; H, 3.58; N, 15.56. Found: C, 58.75; H, 3.56; N, 15.59.

5-((10H-Phenothiazin-10yl)methyl)-4-(4-fluorobenzylideneamino)-4H1,2,4-triazole-3-thiol (6c)

Yield 72\%; m.p. $160-162{ }^{\circ} \mathrm{C}$; IR (KBr)v $\left(\mathrm{cm}^{-1}\right)$ : $2890(\mathrm{CH}), 2760(\mathrm{SH})$, $1658(\mathrm{C}=\mathrm{O}), 1572(\mathrm{C}=\mathrm{N}) 1195(\mathrm{C}-\mathrm{F}) ;{ }^{1} \mathrm{H}$ NMR (DMSO-d, $\left.400 \mathrm{MHz}\right) \delta$ : $12.42(\mathrm{~s}, 1 \mathrm{H}, \mathrm{SH}), 9.10(\mathrm{~s}, 1 \mathrm{H}), 7.67(\mathrm{dd}, 2 \mathrm{H}, \mathrm{Ar}-\mathrm{H}, J=8.0,2.40 \mathrm{~Hz}), 7.23$ $(\mathrm{dd}, 2 \mathrm{H}, J=8.06,2.40, \mathrm{Ar}-\mathrm{H}), 7.20(\mathrm{dd}, 2 \mathrm{H}, J=8.0,2.40, \mathrm{Ar}-\mathrm{H}), 7.14(\mathrm{dd}, 2 \mathrm{H}$, $\mathrm{Ar}-\mathrm{H}, J=7.94,2.38 \mathrm{~Hz}), 7.08$ (t, $2 \mathrm{H}, J=8.50, \mathrm{Ar}-\mathrm{H}), 7.04(\mathrm{dd}, 2 \mathrm{H}, J=7.84$, $2.12 \mathrm{~Hz}$ ), $5.12\left(\mathrm{~s}, 2 \mathrm{H}, \mathrm{NCH}_{2}\right) ;{ }^{13} \mathrm{C} \mathrm{NMR}\left(\mathrm{DMSO}-d_{6}, 100 \mathrm{MHz}\right) \delta: 165.50(\mathrm{~d}$ $J=248.20), 161.20,154.20,147.28,144.30(2 \mathrm{C}), 130.70(2 \mathrm{C}), 128.10(2 \mathrm{C})$, $127.90,127.15$ (2C), 123.30 (2C), 121.60 (2C), 116.90 (2C), 115.50 (d, J=18 $\mathrm{Hz}, 2 \mathrm{C}), 54.14 ; \mathrm{LC}-\mathrm{MS}(70 \mathrm{eV}): \mathrm{m} / \mathrm{z}=433.08$. Anal. Calcd. for $\mathrm{C}_{22} \mathrm{H}_{16} \mathrm{FN}_{5} \mathrm{~S}_{2}$ : C, 60.95; H, 3.72; N, 16.15. Found: C, 60.98; H, 3.75; N, 16.17.

5-((10H-Phenothiazin-10yl)methyl)-4-(4-methoxybenzylideneamino)4H-1,2,4-triazole-3-thiol (6d)

Yield 68\%; m.p. $218-220{ }^{\circ} \mathrm{C}$; IR (KBr)v $\left(\mathrm{cm}^{-1}\right)$ : $2893(\mathrm{CH}), 2768(\mathrm{SH})$, $1650(\mathrm{C}=\mathrm{O}), 1574(\mathrm{C}=\mathrm{N}) ;{ }^{1} \mathrm{H}$ NMR (DMSO-d, $\left.400 \mathrm{MHz}\right) \delta: 13.10(\mathrm{~s}, 1 \mathrm{H}$, $\mathrm{SH}), 9.16(\mathrm{~s}, 1 \mathrm{H}), 7.90$ (dd, 2H, Ar-H, $J=7.98,2.45 \mathrm{~Hz}), 7.51$ (dd, 2H, $J=$ $8.00,2.44$, Ar-H), $7.24(\mathrm{~m}, 4 \mathrm{H}, \mathrm{Ar}-\mathrm{H}), 7.14$ (dd, $2 \mathrm{H}, \mathrm{Ar}-\mathrm{H}, J=7.96,2.42$ $\mathrm{Hz}), 7.00\left(\mathrm{dd}, 2 \mathrm{H}, J=7.84,2.10 \mathrm{~Hz}\right.$ ), $5.24\left(\mathrm{~s}, 2 \mathrm{H}, \mathrm{NCH}_{2}\right), 3.82(\mathrm{~s}, 3 \mathrm{H}, \mathrm{CH} 3)$; ${ }^{13} \mathrm{C}$ NMR (DMSO- $\left.d, 100 \mathrm{MHz}\right) \delta: 165.15,160.10,158.20,154.24,144.38$ (2C), 130.40 (2C), 127.70 (2C), 127.23 (2C), 125.12, 123.18 (2C), 122.46 (2C), 115.40 (2C), 114.20 (2C), 55.20 (CH3), 55.14; LC-MS (70 eV): m/z = 445.10. Anal. Calcd. for $\mathrm{C}_{22} \mathrm{H}_{16} \mathrm{ClN}_{5} \mathrm{~S}_{2}$ : C, 62.00; H, 4.30; N, 15.72. Found: $\mathrm{C}$, 61.98; H, 4.33; N, 15.70 .

5-((10H-Phenothiazin-10yl)methyl)-4-(4-hydroxybenzylideneamino)4H-1,2,4-triazole-3-thiol (6e)

Yield 75\%; m.p. $214-216^{\circ} \mathrm{C}$; IR (KBr)v $\left(\mathrm{cm}^{-1}\right)$ : $3320(\mathrm{O}-\mathrm{H}), 2888(\mathrm{CH})$, $2758(\mathrm{SH}), 1656(\mathrm{C}=\mathrm{O}), 1578(\mathrm{C}=\mathrm{N}) ;{ }^{1} \mathrm{H}$ NMR $(\mathrm{DMSO}-d, 400 \mathrm{MHz}) \delta: 13.12$ (s, 1H, SH), $9.90(\mathrm{~s}, \mathrm{O}-\mathrm{H}), 9.16(\mathrm{~s}, 1 \mathrm{H}), 7.80(\mathrm{dd}, J=7.98,2.45 \mathrm{~Hz}, 2 \mathrm{H}, \mathrm{Ar}-\mathrm{H})$, 7.22 (dd, 2H, Ar-H, $J=8.10,2.44 \mathrm{~Hz}$ ), 7.20 (dd, 2H, Ar-H, $J=7.94,2.46$ $\mathrm{Hz}), 7.10(\mathrm{dd}, 2 \mathrm{H}, J=7.82,2.20 \mathrm{~Hz}), 6.90(\mathrm{~m}, 4 \mathrm{H}, \mathrm{Ar}-\mathrm{H}), 5.24\left(\mathrm{~s}, 2 \mathrm{H}, \mathrm{NCH}_{2}\right)$; ${ }^{13} \mathrm{C}$ NMR (DMSO- $\left.d, 100 \mathrm{MHz}\right) \delta: 165.00,161.10,158.20,154.24,144.98$ (2C), 130.30 (2C), 127.80 (2C), 126.90 (2C), $124.92,123.16$ (2C), 122.26 (2C), 116.10 (2C), 115.00 (2C), 53.80; LC-MS (70 eV): $\mathrm{m} / \mathrm{z}=431.01$. Anal. Calcd. for $\mathrm{C}_{22} \mathrm{H}_{16} \mathrm{ClN}_{5} \mathrm{~S}_{2}$ : C, 61.23; H, 3.97; N, 16.23. Found: C, 61.21; H, 4.00; $\mathrm{N}, 16.25$.
5-((10H-Phenothiazin-10yl)methyl)-4-(2,4-dichlorobenzylideneamino)4H-1,2,4-triazole-3-thiol (6f)

Yield 75\%; m.p. $230-232{ }^{\circ} \mathrm{C}$; IR (KBr)v $\left(\mathrm{cm}^{-1}\right)$ : $2893(\mathrm{CH}), 2768(\mathrm{SH})$, $1650(\mathrm{C}=\mathrm{O}), 1574(\mathrm{C}=\mathrm{N}) 682(\mathrm{C}-\mathrm{Cl}) ;{ }^{1} \mathrm{H}$ NMR (DMSO-d, $\left.400 \mathrm{MHz}\right) \delta$ : $13.12(\mathrm{~s}, 1 \mathrm{H}, \mathrm{SH}), 9.18(\mathrm{~s}, 1 \mathrm{H}), 7.92(\mathrm{~d}, \mathrm{Ar}-\mathrm{H}, J=7.98 \mathrm{~Hz}), 7.72(\mathrm{~s}, \mathrm{Ar}-\mathrm{H})$, $7.38(\mathrm{~d}, \mathrm{Ar}-\mathrm{H}, J=8.10 \mathrm{~Hz}), 7.20(\mathrm{~m}, 4 \mathrm{H}, \mathrm{Ar}-\mathrm{H}), 7.10(\mathrm{dd}, 2 \mathrm{H}, \mathrm{Ar}-\mathrm{H}, J=$ $7.96,2.38 \mathrm{~Hz}) 6.95(\mathrm{dd}, 2 \mathrm{H}, J=7.88,2.15 \mathrm{~Hz}), 5.24\left(\mathrm{~s}, 2 \mathrm{H}, \mathrm{NCH}_{2}\right) ;{ }^{13} \mathrm{C} \mathrm{NMR}$ (DMSO-d , $100 \mathrm{MHz}) \delta: 163.15,158.30,154.24,144.88$ (2C), 131.40, 131.10, 129.00, 129.60, 128.50 (2C), 128.10, 127.50 (2C), 127.10, 123.14 (2C), 122.40 (2C), 115.20 (2C), 55.18; LC-MS $(70 \mathrm{eV}): \mathrm{m} / \mathrm{z}=483.01$. Anal. Calcd. for $\mathrm{C}_{22} \mathrm{H}_{15} \mathrm{Cl}_{2} \mathrm{~N}_{5} \mathrm{~S}_{2}$ : C, 54.55; H, 3.12; N, 14.46. Found: C, 54.57; H, 3.15; N, 14.48.

5-((10H-Phenothiazin-10yl)methyl)-4-(2,5-dibromobenzylideneamino)4H-1,2,4-triazole-3-thiol (6g)

Yield 81\%; m.p. $228-230{ }^{\circ} \mathrm{C}$; IR (KBr)v $\left(\mathrm{cm}^{-1}\right)$ : $2892(\mathrm{CH}), 2761(\mathrm{SH})$, $1654(\mathrm{C}=\mathrm{O}), 1570(\mathrm{C}=\mathrm{N}) 726(\mathrm{C}-\mathrm{Br}) ;{ }^{1} \mathrm{H}$ NMR $\left(\mathrm{DMSO}-d_{6}, 400 \mathrm{MHz}\right) \delta: 12.50$ (s, 1H, SH), $9.30(\mathrm{~s}, 1 \mathrm{H}), 7.92(\mathrm{~s}, \mathrm{Ar}-\mathrm{H}), 7.60(\mathrm{~d}, J=8.05, \mathrm{Ar}-\mathrm{H}), 7.55$ (d, $J=8.10, \mathrm{Ar}-\mathrm{H}), 7.22$ (dd, $J=8.20,2.40,2 \mathrm{H}, \mathrm{Ar}-\mathrm{H}), 7.20(\mathrm{~d}, J=8.10,2 \mathrm{H}$, Ar-H ), 7.12 (dd, 2H, Ar-H, $J=7.98,2.36 \mathrm{~Hz}$ ), 7.00 (dd, $2 \mathrm{H}, J=7.80,2.30$ $\mathrm{Hz}), 5.22\left(\mathrm{~s}, 2 \mathrm{H}, \mathrm{NCH}_{2}\right) ;{ }^{13} \mathrm{C}$ NMR (DMSO- $\left.d, 100 \mathrm{MHz}\right) \delta: 165.10,158.18$, $154.20,144.12$ (2C), 134.00, 133.20, 132.90, 130.30, 128.80 (2C), 127.60 (2C), 125.10, 124.16, 123.22 (2C), 122.10(2C), 115.66 (2C), 55.14; LC-MS $(70 \mathrm{eV}): \mathrm{m} / \mathrm{z}=572.91$. Anal. Calcd. for $\mathrm{C}_{22} \mathrm{H}_{16} \mathrm{Br}_{2} \mathrm{~N}_{5} \mathrm{~S}_{2}: \mathrm{C}, 46.09 ; \mathrm{H}, 2.64 ; \mathrm{N}$, 12.22. Found: C, 46.07; H, 2.61; N, 12.25 .

5-((10H-Phenothiazin-10yl)methyl)-4-(3,5-difluorobenzylideneamino)4H-1,2,4-triazole-3-thiol (6h)

Yield 68\%; m.p. $150-152{ }^{\circ} \mathrm{C}$; IR $(\mathrm{KBr}) v\left(\mathrm{~cm}^{-1}\right)$ : $2892(\mathrm{CH}), 2766(\mathrm{SH})$, $1656(\mathrm{C}=\mathrm{O}), 1572(\mathrm{C}=\mathrm{N}) 1190(\mathrm{C}-\mathrm{F}) ;{ }^{1} \mathrm{H}$ NMR (DMSO-d, $\left.400 \mathrm{MHz}\right) \delta$ : $12.80(\mathrm{~s}, 1 \mathrm{H}, \mathrm{SH}), 9.08(\mathrm{~s}, 1 \mathrm{H}), 7.50(\mathrm{~d}, 2 \mathrm{H}, \mathrm{Ar}-\mathrm{H}, J=7.50 \mathrm{~Hz}), 7.20(\mathrm{~m}$, $4 \mathrm{H}, \mathrm{Ar}-\mathrm{H}), 7.10$ (dd, 2H, Ar-H, $J=7.98,2.30 \mathrm{~Hz}), 7.00(\mathrm{dd}, 2 \mathrm{H}, J=7.88$, $2.22 \mathrm{~Hz}$ ), $6.95(\mathrm{t}, J=7.5, \mathrm{Ar}-\mathrm{H}), 5.14\left(\mathrm{~s}, 2 \mathrm{H}, \mathrm{NCH}_{2}\right) ;{ }^{13} \mathrm{C} \mathrm{NMR}$ (DMSO- $d$, $100 \mathrm{MHz}) \delta: 163.80(\mathrm{~d}, J=246.80,2 \mathrm{C}-\mathrm{F}), 161.10,154.40,147.28,144.60$ (2C), 134.70, 128.20(2C), 127.15 (2C), 123.10 (2C), 122.60 (2C), $115.90(2 \mathrm{C})$, $110.50(\mathrm{~d}, 2 \mathrm{C}), 104.50,54.16 ; \mathrm{LC}-\mathrm{MS}(70 \mathrm{eV}): \mathrm{m} / \mathrm{z}=451.07$. Anal. Calcd. for $\mathrm{C}_{22} \mathrm{H}_{16} \mathrm{~F}_{2} \mathrm{~N}_{5} \mathrm{~S}_{2}$ : C, 58.52; H, 3.35; N, 15.51. Found: C, 58.54; H, 3.37; N, 15.54 .

5 - ( ( $10 \mathrm{H}-\mathrm{Ph}$ e nothiazin-10yl) methyl) - 4 - ( 3, 5 dimethoxybenzylideneamino)-4H-1,2,4-triazole-3-thiol (6i)

Yield 70\%; m.p. 239-240 ${ }^{\circ} \mathrm{C}$; IR (KBr)v $\left(\mathrm{cm}^{-1}\right)$ : $2898(\mathrm{CH}), 2770(\mathrm{SH})$, $1658(\mathrm{C}=\mathrm{O}), 1578(\mathrm{C}=\mathrm{N}) ;{ }^{1} \mathrm{H}$ NMR $\left(\mathrm{DMSO}-d_{6}, 400 \mathrm{MHz}\right) \delta: 13.48(\mathrm{~s}, 1 \mathrm{H}$, $\mathrm{SH}), 9.26(\mathrm{~s}, 1 \mathrm{H}), 7.22(\mathrm{dd}, 2 \mathrm{H}, \mathrm{Ar}-\mathrm{H}, J=7.90,2.40 \mathrm{~Hz}), 7.20(\mathrm{dd}, 2 \mathrm{H}, J=$ 8.10, 2.44, Ar-H), 7.14 (d, $J=8.00,2 \mathrm{H}, \mathrm{Ar}-\mathrm{H}), 7.00$ (dd, 2H, Ar-H, $J=8.18$, $2.46 \mathrm{~Hz}), 7.00(\mathrm{dd}, 2 \mathrm{H}, J=7.84,2.10 \mathrm{~Hz}), 6.90(\mathrm{~s}, \mathrm{Ar}-\mathrm{H}), 5.20\left(\mathrm{~s}, 2 \mathrm{H}, \mathrm{NCH}_{2}\right)$, 3.84 (s, 6H, CH3); ${ }^{13} \mathrm{C}$ NMR (DMSO-d, $\left.100 \mathrm{MHz}\right) \delta: 164.15$ (2C), 160.15, $154.24,148.50,144.20$ (2C), 134.40, 127.80 (2C), $127.40(2 \mathrm{C}), 123.22(2 \mathrm{C})$, 122.44 (2C), 115.48 (2C), 104.20 (2C), 103.50, 55.40 (2CH3), 55.14; LC-MS $(70 \mathrm{eV}): \mathrm{m} / \mathrm{z}=475.11$. Anal. Calcd. for $\mathrm{C}_{24} \mathrm{H}_{21} \mathrm{~N}_{5} \mathrm{O}_{2} \mathrm{~S}_{2}: \mathrm{C}, 60.61 ; \mathrm{H}, 4.45 ; \mathrm{N}$, 14.73. Found: C, 60.63; H, 4.42; N, 14.75

\section{PHARMACOLOGICAL SCREENING}

Antioxidant screening in vitro

The compounds $\mathbf{6 a - i}$ were evaluated for antioxidant activity using the Nitric oxide (NO), Hydrogen peroxide $\left(\mathrm{H}_{2} \mathrm{O}_{2}\right)$ and 1,1-Diphenyl-1-picrylhydrazyl (DPPH) methods.

\section{NO scavenging activity}

NO biological activity was determined using the methods by Green et al. and Marcocci et al. with slight modification ${ }^{46,47}$. Briefly, NO radicals were first created with the sodium nitroprusside. Sodium nitroprusside $(10 \mathrm{mmol}, 1 \mathrm{~mL})$, phosphate buffer saline (PBS) $(0.25 \mathrm{mmol}, 1.5 \mathrm{~mL}, \mathrm{pH} 7.5)$ were added to the various amounts of experimental compounds $(25,50,75$ and $100 \mu \mathrm{g} / \mathrm{mL})$ and incubated at $25{ }^{\circ} \mathrm{C}$ for $2.5 \mathrm{~h}$. Next, the reaction mixtures $(1 \mathrm{~mL})$ were preserved with $1 \mathrm{~mL}$ of Griess reagent. All experiments were performed in triplicates. The absorbance of the samples was measured at $546 \mathrm{~nm}$ using the chromatophore and NO activity was calculated by the following formula

Scavenging activity $(\%)=[($ A control-A sample $) /$ A control $]$ X 100

A control = Absorbance of the control reaction (all reagents without the test compounds)

A sample $=$ Absorbance of the test compound.

$\mathrm{H}_{2} \mathrm{O}_{2}$ scavenging activity

The compounds $\mathrm{H}_{2} \mathrm{O}_{2}$ biological activity was determined using the previous method $^{48}$. A solution of $\mathrm{H}_{2} \mathrm{O}_{2}(10 \mathrm{mmol})$ in PBS $(0.25 \mathrm{mmol})$ was prepared and various amounts of test compounds as described in above section were added to the PBS containing $\mathrm{H}_{2} \mathrm{O}_{2}(4 \mathrm{~mL})$. The absorbance of the compounds was recorded at $230 \mathrm{~nm}$, percent of $\mathrm{H}_{2} \mathrm{O}_{2}$ scavenging activity was calculated by using the above mentioned formula. All the tests were carried out in triplicate. 


\section{DPPH scavenging activity}

The compounds ability to donate electrons or hydrogen atoms was evaluated by the DPPH colorimetric method based on the decolorization of purple methanol solution ${ }^{49}$. This assay produces the DPPH stable radicals. Various concentrations of compounds (refer to above sections) in methanol $(1 \mathrm{~mL})$ were added to the $4 \mathrm{~mL}$ of DPPH methanol solution $(0.004 \%(\mathrm{w} / \mathrm{s})$ and incubated for $0.5 \mathrm{~h}$ at $25^{\circ} \mathrm{C}$. After this incubation period, the absorbance was measured at $517 \mathrm{~nm}$. The percentage of DPPH scavenging activity was calculated using the above equation.

\section{ACKNOWLEDGEMENT}

The authors acknowledge to the National Research Foundation of South Africa, University of KwaZulu-Natal, Durban, South Africa and Department of Chemistry, Annamacharya Institute of Technology \& Sciences, Tirupati, India for the facilities.

\section{REFERENCES}

1. A. P. Ghose, V. N. Viswanadhan, J. J. Wendoloski, Comb. Chem. 1(1), 55, (1999).

2. A. F. Pozharskii, A. T. Soldatenkov, A. R. Katritzky, John Wiley \& Sons (1997).

3. R. E. Looper, M. T. C. Runnegar, R. M. Williams, Angewandte Chemie International Edition. 44(25), 3879, (2005).

4. I. Khan, S. Ali, S. Hameed, N. H. Rama, M. T. Hussain, A. W. R. Uddin, Z. Ul-Haq, A. Khan, S. Ali, I. Choudhary, Eur. J. Med. Chem. 45, 5200, (2010).

5. W. A. Bayoumi, M. A. Elsayed, H. N. Baraka, L. Abou-zeid, Arch. Pharm. Chem. Life Sci. 345, 902, (2012).

6. H. Adibi, A. Rashidi, M. M. Khodaei, A. Alizadeh, M. B. Majnooni, N. Pakravan, R. Abiri, D. Nematollahi, Chem. Pharm. Bull. 59(9), 1149, (2011).

7. L. F. Yamaguchi, J. H. Lago, T. M. Tanizak, P. Dimassio, M. J. Kato, J. Photochem. 16, 1838, (2006).

8. N. M. A. El-Ebiary, R. H. Swellem, H. M. Abdel-Tawab, G. A. M. Nawwar, Arch. Pharm. Chem. Life Sci. 9, 528, (2010).

9. P. Xu, B. Yu, F.L. Li, X. F. Cai, C. Q. Ma, Trends Microbiol . 14(9), 398, (2006).

10. A. Jaszczyszyn, K. Gasiorowski, P. Swiatek, W. Malinka, K. CieslikBoczula, J. Petrus, B. Czarnik-Matusewicz, Pharmacol. Reports. 64, 16, (2012).

11. K. Nilgun, A. Idil, O. Sumru, G. Aysel, U. D. Sonmez, E. Aylin, O. Osman, Arch. Pharm. Pharm. Med. Chem. 332, 422, (1999).

12. S. D. Srivastava, P. Kohli, Proc. Natl. acad. Sci. India. 77(A), 199, (2007).

13. M. Mayer, T. Lang, S. Gerber, P. B. Madrid, I. G. Pinto, R. K. Guy, T. L. James, Chem. Biol. 13, 993, (2006).

14. Y. S. Sadanandam, M. M. Shetty, A. B. Rao, Y. Rambabu, Eur. J. Med. Chem. 44, 197, (2009).

15. S. Maddila, S. Gorle, M. Singh, P. Lavanya, S. B. Jonnalagadda, Lett. Drug Desig. Discov. 10(10), 977, (2013).

16. T. Kurihara, N. Motohashi, H. Sakagami, H. Molnar, J. Anticancer Res. 19, 4081, (1999).

17. T. Kurihara, K. Nojima, H. Sakagami, N. Motohashi, H. Molnar, J. Anticancer Res. 19, 3895, (1999).

18. O. W. Foye, "Principles of Medicinal Chemistry" 3rd, Lippincott Williams \& Wilkins Publishers. (1996).

19. A. D. Mosnaim, V. V. Ranade, M. E. Wel, J. Puente, A. M. Valenzuela, Am. J. Ther. 13, 261, (2006).

20. P. B. Madrid, W. E. Polgar, L. Toll, M. J. Tanga, Bioorg. Med. Chem. Lett. 17, 3014, (2007)

21. P. Archana, K. Rani, V. K. Bajajsrivastava, R. Chandra, K. Kumar, Drug. Res. 53, 301, (2003)

22. K. V. Tandon, H. K. Maurya, A. Tripathi, G. B. Shivakeshava, P. K. Shukla, P. Srivastava, D. Panda, Eur. J. Med. Chem. 44, 1086, (2009).

23. L. Wei, A. Amar, A. Marine, C. Li, A. Jean-Marie, The Journal of Immunology. 167, 2929, (2001).

24. A. R. Prasad, T. Ramalingam, A. B. Rao, P. V. Diwan, P. B. Sattur, Eur. J. Med. Chem. 24, 199, (1989).

25. N. Singhal, P. K. Sharma, R. Dudhe, N. Kumar, J. Chem. Pharm. Res. 3(2), 126, (2011).

26. S. Banerjee, S. Ganguly, K. K. Sen, J. Adv. Phar. Edu. \& Res. 3, 102, (2013).
24. S. Maddila, P. Ramakanth, S. B. Jonnalagadda, Lett. Org. Chem. 10(10), 693, (2013).

28. S. Maddila, S. B. Jonnagadda, Pharma. Chem. J. 46(11), 1, (2013).

29. S. Maddila, A. S. Kumar, S. Gorle, M. Singh, P. Lavanya, S. B. Jonnalagadda, Lett. Drug Desig. \& Discov. 10(2), 186, (2013).

30. S. Maddila, S. B. Jonnalagadda, Lett. Drug Desig. \& Discov. 9(7), 687, (2012).

31. S. Maddila, P. Ramakanth, S. B. Jonnalagadda, J. Heter. Chem. (MS ID: JHET-13-0053.R1-In Press) (2013).

32. R. Kharb, P. C. Sharma, M. S. Yar, J. Enz. Inhib. Med. Chem. 26(1), 1, (2011).

33. Y. A. Al-Soud, M. N. Al-Dweri, N. A. Al-Masoudi, IL Farmaco. 59, 775, (2004).

34. A. Assandri, A. Omodei-Sale, P. Ferrari, G. Tuan, P. Perazzi, A. Ripamonti, E. Martinelli, Xenobiotica. 12(1), 19, (1982).

35. A. Almasirad, S. A. Tabatabai, M. Faizi, A. Kebriaeezadeh, N. Mehrabi, A. Dalvandia, A. Shafiee, Bioorg. Med.Chem. Lett. 14, 6057, (2004).

36. K. Ilkay, K. Guniz, R. Sevim, O-S. Gulten, O. Osman, B. Ibrahim, A. Tuncay, P. James, IL Farmaco. 59, 893, (2004).

37. Y. A. Al-Soud, N. A. Al-Masoudi, A. S. F. El-Rahman, Bioorg. Med. Chem. 11, 1701, (2003).

38. S. S. Parmar, A. K. Gupta, H. H. Singh, T. K. Gupta, J. Med. Chem. 15(9), 999, (1972)

39. S. Maddila, P. Lavanya, D. Sudhakar, K. Vasu, C. V. Rao, J. Chem Pharm. Res. 2 (1), 497, (2010).

40. S. Maddila, P. Lavanya, K. N. Raju, S. B. Jonnalagadda, C. V. Rao, Org. Commun. 4(2), 33, (2011).

41. S. Maddila, S. Gorle, N. Seshadri, P. Lavanya, S. B. Jonnalagadda, DOI: 10.1016/j.arabjc.2013.04.003, In-press - (2013).

42. S. Maddila, S. B. Jonnalagadda. Archive Der Phar. Life Sci. 345, 163 (2012).

43. P. Lavanya, S. Maddila, S. B. Jonnalagadda, C.V. Rao. Asian J. Chem. 25(1), 385, (2013)

44. A. Upadhyay, S. K. Srivastava, S. D. Srivastava, Jordon J. Chem. 5(1), 1, (2010).

45. S. D. Srivastava, P. Kohli, Proceed. Nat. Aca. Sci. India, Sec. B: Biol. Sci. 77(3), 199, (2007).

46. P. R. Murray, E. J. Baron, M. A. Pfaller, F. C. Tenover, R. H. Yolken, G. L. Wood, Washington (Eds.), Am. Soc. Microbiol. Washington DC, (1995).

47. S. Maddila, S. B. Jonnalagadda. Lett. Org. Chem. 10, 374, (2013).

48. R. J. Ruch, S. J. Cheng, J. E. Klaunig, Carcinogenesis. 10, 1003, (1989).

49. M. Burits, F. Bucar, Phytother Res. 14, 323, (2000). 